\title{
Effect of laser pulse width on wave packet motion in femotosecond pump-probe pulses
}

Wei Guo ${ }^{\mathrm{a}, \mathrm{b} *}$, Xiaojing Feng ${ }^{\mathrm{a}}$, Xingqiang $\mathbf{L U}^{\mathrm{c}}$,

${ }^{a}$ School of Electrical Engineering, University of South China, Hengyang 421001, China

${ }^{b}$ State Key Laboratory of Molecular Reaction Dynamics, Dalian Institute of Chemical Physics, Dalian 116023, China

${ }^{c}$ School of Nuclear Science and Technology, University of South China, Hengyang 421001, China

\section{ABSTRACT}

The dependence of the wave packet process of three-level ladder $\mathrm{K}_{2}$ molecules on pulse width was investigated via time-dependent wave packet method, and was firstly quantified. There is no Autler-Townes splitting in the photoelectron spectrum with weaker pump intensity or shorter pulse width. The oscillation period of wave packet increases and the oscillation amplitude decreases with increasing pulse width. The pulse width affects Rabi oscillation, which results in the periodical change of the populations of the electronic state. Quantifying its effect on the population of the excited state shows that the change frequency varies with increasing pump intensity. The results illustrates that the needed population in electronic state of interested can be obtained by controlling pulse width, which provide some important basis for light manipulation of molecular processes experimentally.

Keywords:

Intense femtosecond pump-probe pulses; Wave packet motion; Time-dependent wave packet approach; Photoelectron spectra; State populations

\footnotetext{
${ }^{*}$ Corresponding author.

E-mail address: vella99@163.com (W. Guo).
} 


\section{Introduction}

With the development of ultrashort and ultrastrong laser pulse technology, more and more researches have focused on the control of molecular dynamics in real time. Laser parameters, such as laser intensity[1-19], laser wavelength[4-8,12-16,18], pulse width[1-3,7-10,18,19], pulse envelope[4,5], and delay time [10-17] can be used for controlling the excitation, ionization and dissociation processes of molecules, and have been studied in multi-level molecular systems.

For a diatomic molecule, especially an alkali metal dimer, the wave packet dynamics of the excited state can be studied by using femtosecond pump-probe technique. Christoph et al. [1] and Hu et al. [2,3] studied the effect of laser intensity and pulse width on photoelectron spectra of four-level $\mathrm{Na}_{2} / \mathrm{Li}_{2}$, and suggested that there is no Autler-Townes (AT) triple splitting for weaker pump laser intensity or shorter pulse width, longer pulse width can induce symmetric AT triple splitting. Yao et al. [4], Qin et al. [5] and Guo et al. [6] suggested the laser intensity, pulse width and laser wavelength affect the asymmetry of AT splitting, and can control the selective population of dresses states of four-level $\mathrm{K}_{2}$. Sun et al. [7] and Yuan et al. [8] studied the effect of laser intensity, pulse width, molecular rotation and molecular alignment on photoelectron spectra of three-level $\mathrm{Na}_{2}$, and suggested that there is no AT double splitting for weaker laser intensity or shorter pulse width, longer pulse width can induce symmetric AT double splitting. Yuan et al. $[9,10]$ indicated that the scheme with short pulse width and positive delay time is more effective for controlling the wave packet dynamics and the population transfer of three-level $\mathrm{Na}_{2}$, which is explained using the light-induced potentials (LIPs). Liu et al. [11,12] proposed that asymmetric double peaks arise for longer delay time, the delay time only alters the peak height, but does not alter the peak positions of photoelectron spectra of three-level $\mathrm{Li}_{2}$. Han et al. [13] suggested there is no AT splitting for weaker pump intensity or longer delay time, the pump wavelength detuning induced the asymmetric AT double splitting for three-level $\mathrm{Li}_{2}$. Yao et al. [14,15] proposed that the laser intensity, pump wavelength and delay time can control the selective population of 
dressed states of three-level $\mathrm{K}_{2}$. Guo et al. [16] studied the noadiabatic effect of NaI. Zhang et al. [17] suggested that the laser wavelength affects the oscillation period of wave packet, the delay time alters the peak positions and height of photoelectron spectra of NaLi. Wang et al. [18] and Meng et al. [19] studied the effect of the laser intensity, laser wavelength, pulse width and delay time on state populations of NO, and suggested the periodical change with laser intensity and pulse width.

The AT symmetric/asymmetric double/triple splitting and the selective population of dressed states of $\mathrm{K}_{2}$ molecule may be attained by altering the laser intensity, laser wavelength and pulse envelope $[4-6,14,15]$. No studies focus on the effect of laser pulse width on wave packet dynamics. This paper presents new data on the pulse width dependence of the photoelectron spectra and state populations in three-level ladder $\mathrm{K}_{2}$ molecule driven by pump-probe pulses via time-dependent quantum wave packet method.

\section{Theory}

Three electronic states, ground state $|X\rangle\left(|X\rangle^{1} \Sigma_{g}^{+}\right)$, excited state $|B\rangle\left(|B\rangle^{1} \Sigma_{g}^{+}\right)$ and ion ground state $\left|X^{+}\right\rangle\left(\left|X^{+}\right\rangle^{2} \Sigma_{g}^{+}\right)$are involved in the multiphoton ionization of $\mathrm{K}_{2}$ molecule $[14,15,20]$. The potential energy curves are shown in Fig. 1. The transition from $|X\rangle$ to $|B\rangle$ is excited by resonant one-photon absorption, then the populations in $|B\rangle$ are ionized through single-photon absorption after the delay time, and the emitted photoelectron is detected from $\left|X^{+}\right\rangle$. 


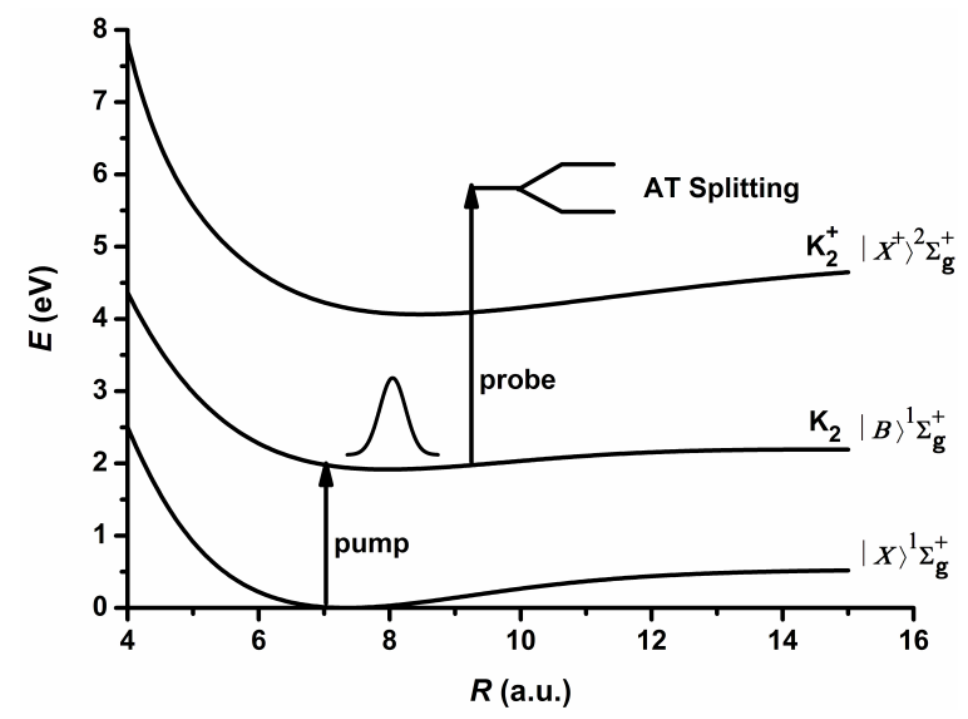

Fig. 1. Potential energy curves of $\mathrm{K}_{2}$ molecule.

For the three-state model, the wave functions can be written in the column vector $\boldsymbol{\Psi}=\left(\psi_{X}, \psi_{B}, \psi_{I}\right)^{T}$,

where $\psi_{X}, \psi_{B}$ and $\psi_{I}$ are the wave functions for the states $|X\rangle,|B\rangle$ and $\left|X^{+}\right\rangle$, respectively. The ion ground state $\left|X^{+}\right\rangle$is a continuum state, and is discretized into a band of quasicontinuum states. The $\psi_{I}$ can be further expressed as

$\psi_{I}=\left(\psi^{(1)}, \psi^{(2)}, \ldots, \psi^{(N)}\right)^{T}$

where $N$ is the number of discrete states of $\mathrm{K}_{2}$ ion. In the Born-Oppenheimer frame, the time-dependent Schrödinger equation can be expressed as

$i \hbar \frac{\partial}{\partial t} \boldsymbol{\Psi}=\left(\boldsymbol{T}+\boldsymbol{V}+\boldsymbol{H}^{\prime}\right) \boldsymbol{\Psi}$

The kinetic energy $\boldsymbol{T}$ can be expressed as

$\boldsymbol{T}=-\frac{\hbar^{2}}{2 \mu} \frac{\partial^{2}}{\partial R^{2}}\left(\begin{array}{ccc}1 & 0 & \boldsymbol{\theta} \\ 0 & 1 & \boldsymbol{\theta} \\ \tilde{\boldsymbol{\theta}} & \tilde{\boldsymbol{\theta}} & \Pi\end{array}\right)$,

where $\mu$ is the reduced mass of the nuclei, $R$ is the internuclear distance, $\boldsymbol{\theta}=(0,0, \ldots, 0)$ is a zero vector with $N$-component, $\tilde{\boldsymbol{\theta}}$ is the transverse of the vector $\boldsymbol{\theta}$ and $\Pi$ is a $N \times N$ unit matrix.

The potential $\boldsymbol{V}$ can be expressed as 
$\boldsymbol{V}=\left(\begin{array}{ccc}V_{X} & 0 & \boldsymbol{\theta} \\ 0 & V_{B} & \boldsymbol{\theta} \\ \tilde{\boldsymbol{\theta}} & \tilde{\boldsymbol{\theta}} & \left(V_{I}+\xi^{(k)}\right) \boldsymbol{\Pi}\end{array}\right)$,

where $V_{\mathrm{X}}, V_{\mathrm{B}}$, and $V_{\mathrm{I}}$ are the potential curves of the states $|X\rangle,|B\rangle$, and $\left|X^{+}\right\rangle$respectively. $\xi^{(i)}=(k-1) \Delta \xi(k=1,2, \ldots, N)$ is the energy of the emitted photoelectron.

The interaction $\boldsymbol{H}^{\prime}$ between $\mathrm{K}_{2}$ molecule and laser field is given by

$$
\boldsymbol{H}^{\prime}=\left(\begin{array}{ccc}
0 & W_{X B} & 0 \\
W_{X B} & 0 & \boldsymbol{W}_{B I} \\
0 & \tilde{\boldsymbol{W}}_{B I} & \boldsymbol{\Lambda}
\end{array}\right),
$$

where $\boldsymbol{W}_{B I}=\left(W_{B I}^{(1)}, W_{B I}^{(2)}, \ldots W_{B I}^{(N)}\right)$ is the vector of coupling between $|B\rangle$ and $\left|X^{+}\right\rangle$states via laser field with $N$-component, $\tilde{W}_{B I}$ is the transverse of the vector $\boldsymbol{W}_{B I}$ and $\boldsymbol{\Lambda}$ is the $N \times N$ zero matrix. The coupling between two states via external laser field is given as follows

$$
\begin{aligned}
& W_{X B}=W_{B X}=\mu_{X B}(R) e_{1} f_{1}(t) \cos \left(\omega_{1} t\right), \\
& W_{B I}=\mu_{B I}(R) e_{2} f_{2}(t) \cos \left[\omega_{2}(t-\Delta t)\right], \\
& f_{1}(t)=\exp \left[-4 \ln 2 \cdot(t / \tau)^{2}\right], \\
& f_{2}(t)=\exp \left\{-4 \ln 2 \cdot[(t-\Delta t) / \tau]^{2}\right\},
\end{aligned}
$$

where $\mu_{X B}(R)$ and $\mu_{B I}(R)$ are the transition dipole moments for $|X\rangle \rightarrow|B\rangle$ and $|B\rangle \rightarrow\left|X^{+}\right\rangle, e_{1}$ and $e_{2}$ are the amplitudes of pump and probe laser fields, $\omega_{1}$ and $\omega_{2}$ are the angular frequencies. $f_{1}(t)$ and $f_{2}(t)$ are the envelopes, $\tau$ is the full width at half maximum (FWHM) of the pulse, $\Delta t$ is the delay time between the pump and probe pulses.

The time-dependent Schrödinger equation is solved by split-operator Fourier methods exactly [21,22], once the wave function $\psi_{i}(R, t)$ is defined, the population in 
each electronic state can be written as [1-8,12-19]

$P_{i}(t)=\int\left|\psi_{i}(R, t)\right|^{2} \mathrm{~d} R,(i=X, B, I)$,

The photoelectron spectrum can be calculated as [1-8, 12-16]

$$
P\left(\xi^{(k)}\right)=\lim _{t \rightarrow \infty} \int \mathrm{d} R\left|\psi^{(k)}\left(R, t, \xi^{(k)}\right)\right|^{2},(k=1,2, \ldots, N),
$$

In the calculation, $\xi^{(k)}$ span over $0-1.4 \mathrm{eV}$ and $N$ equals 140.

\section{Results and disscussions}

The effect of the laser pulse width on the photoelectron spectra is considered with delay time 0 fs. Fig. 2 shows the photoelectron spectra for different pulse widths with delay time $0 \mathrm{fs}$. The photoelectron spectra show double splitting at $30 \mathrm{fs}, 60 \mathrm{fs}$ and $80 \mathrm{fs}$, which is AT splitting and arises from the sufficient Rabi oscillation within the resonant region [4-8,13-15]. The pulse area $\Theta=4 \pi$ (the pulse area is defined as $\left.\Theta=4 \frac{1}{\hbar} \mu_{X B} e_{1} \int_{0}^{\infty} f_{1}(t) \mathrm{d} t\right)$ for pump intensity $5 \times 10^{11} \mathrm{~W} / \mathrm{cm}^{2}$, which is in the conditions for the appearance of AT splitting [14,15]. There is no splitting at $10 \mathrm{fs}$, which is consistent with the observations for four-level $\mathrm{Li}_{2}[2,3]$, and is due to the insufficient laser intensity, i.e. the threshold intensity is stronger for shorter pulse width. The threshold pump intensity is $20 \times 10^{11} \mathrm{~W} / \mathrm{cm}^{2}$ at pulse width $10 \mathrm{fs}$ in the calculation. The double splitting is symmetric at $30 \mathrm{fs}$, which has been observed in three-level $\mathrm{Li}_{2}$ [13], $\mathrm{Na}_{2}[7,8]$ and $\mathrm{K}_{2}[14,15]$. The double splitting is unsymmetric at $60 \mathrm{fs}$ and $80 \mathrm{fs}$. The unsymmetric AT splitting for longer pulse width has been observed in three-level $\mathrm{Na}_{2}$ $[7,8]$, four-level $\mathrm{Na}_{2}[1]$ and $\mathrm{Li}_{2}[2,3]$. 


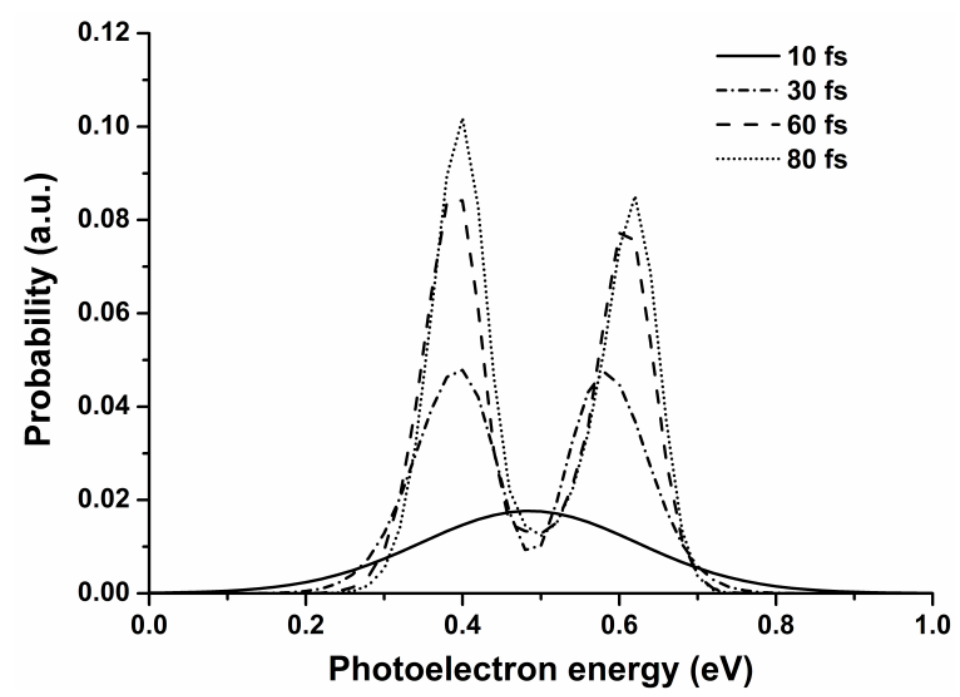

Fig. 2. The photoelectron spectra at different pulse widths. Other laser parameters are delay time $\Delta t=0 \mathrm{fs}$, pump wavelength $\lambda_{1}=640 \mathrm{~nm}$, probe wavelength $\lambda_{2}=460 \mathrm{~nm}$, pump and probe intensity

$$
I_{1}=I_{2}=5.0 \times 10^{11} \mathrm{~W} / \mathrm{cm}^{2} \text {. }
$$

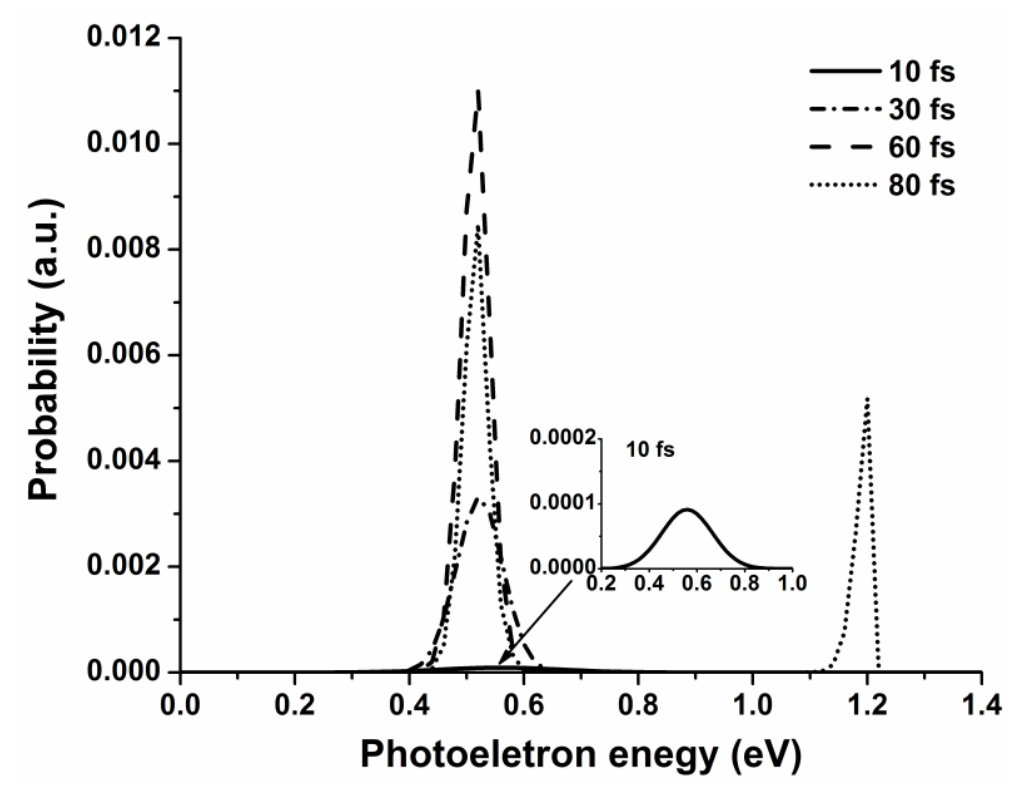

Fig. 3. The photoelectron spectra at different pulse widths. Other laser parameters are delay time $\Delta t=1800 \mathrm{fs}$, pump wavelength $\lambda_{1}=640 \mathrm{~nm}$, probe wavelength $\lambda_{2}=460 \mathrm{~nm}$, pump and probe intensity $I_{1}=I_{2}=5.0 \times 10^{11} \mathrm{~W} / \mathrm{cm}^{2}$.

The effect of the laser pulse width on the photoelectron spectra is considered with delay time 1800 fs. Fig. 3 shows the photoelectron spectra at different pulse widths with delay time 1800 fs. The photoelectron spectra show one peak at10 fs, 30 fs and $60 \mathrm{fs}$, while it is unsymmetric double peaks at $80 \mathrm{fs}$. Liu et al. [11,12] proposed 
the photoelectron spectra show unsymmetric double peaks for longer pulse width and longer delay time (1000 fs-2000 fs).

The energy spread of the single peak in the photoelectron spectra becomes narrower with increasing pulse width. The spread of the kinetic energy spectrum originates from the frequency bandwidth because the bandwidth, which corresponds to the temporal width of the pulse, is associated with the excitation of the molecule from the ground state to a range of continua of the excited states. Hence, in a laser field with a specific peak intensity, the frequency bandwidth become smaller with increasing pulse duration and consequently fewer continuum states can be reached in the excitation, which can lead to a narrower kinetic distribution of the photoelectrons [23].
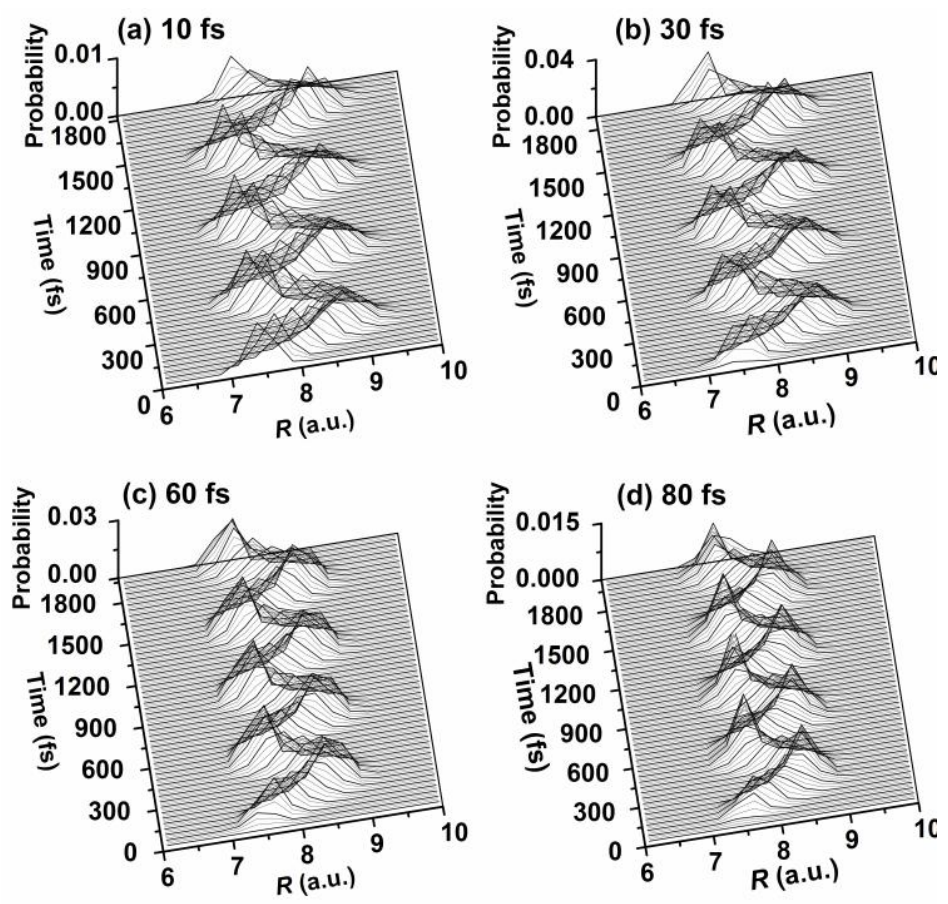

Fig. 4. The evolutions of wave packet in $|B\rangle$ state with time and internuclear distance at different pulse widths. Other laser parameters are the same as those in Fig. 3.

The effect of the pulse width on the wave packet motion is considered. Fig. 4 shows the evolution of wave packet on the excited state $|B\rangle$ with delay time 1800 fs. The wave packet moves between the inner turning point ( 7 a.u.) and the outer tuning point (9.5 a.u.). The periodical wave packet motion was not studied before for $\mathrm{K}_{2}$, but has been observed for three-level $\mathrm{Na}_{2}$ [9] and $\mathrm{NaLi} / \mathrm{NaI}[16,17]$. The oscillation 
period of wave packet increases slightly with increasing pulse width. Due that the pulse width is inversely proportional to the range of energy, the propagation velocity of the molecule on the excited state decreases with increasing pulse width, which results in increasing time for wave packet motion between the inner turning point and the outer tuning point, i.e. the oscillation period increases. The oscillation amplitude decreases with increasing pulse width, which is a consequence of ultrashort excitation and rapid change of the LIPs [9]. The oscillation amplitude is related to the change rate of the LIPs, which is closely associated with pulse widths. The pulse widths get shorter, the LIPs change more rapidly, which results in the decreasing oscillation amplitude with increasing pulse width [9].
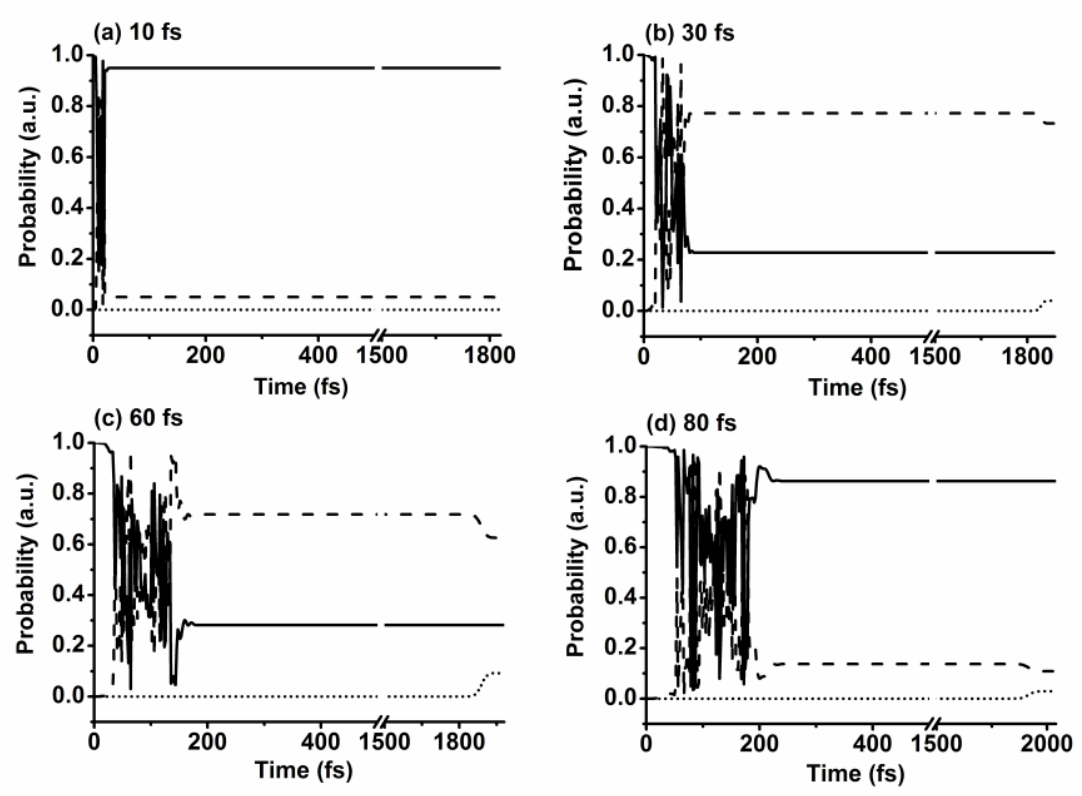

Fig. 5. The evolution of state populations at different pulse widths. The thin solid, dashed and dotted lines denote the populations on ground state $|X\rangle$, excited state $|B\rangle$ and ionic state $\left|X^{+}\right\rangle$, respectively . Other laser parameters are the same as those in Fig. 3.

The effect of the pulse width on state populations is considered. Fig. 5 shows the evolution of state populations at different pulse widths. The Rabi oscillation can be observed when the pump pulse is present. The longer the pulse width is, the longer the oscillation time is. The populations of the ground state and the excited state change periodically with increasing pulse width, which has been observed for NO molecule 


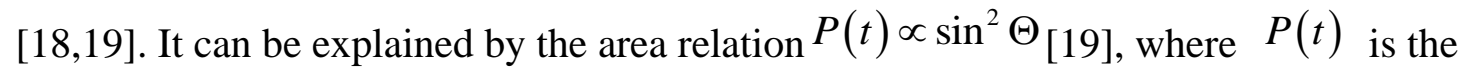
excitation probability, pulse area $\Theta=4 \frac{1}{\hbar} \mu_{X B} e_{1} \int_{0}^{\infty} f_{1}(t) \mathrm{d} t$ is proportional to the pulse width $\tau$ [4], so pulse width can lead to the above change of the population of the excited state by changing pulse area[19].

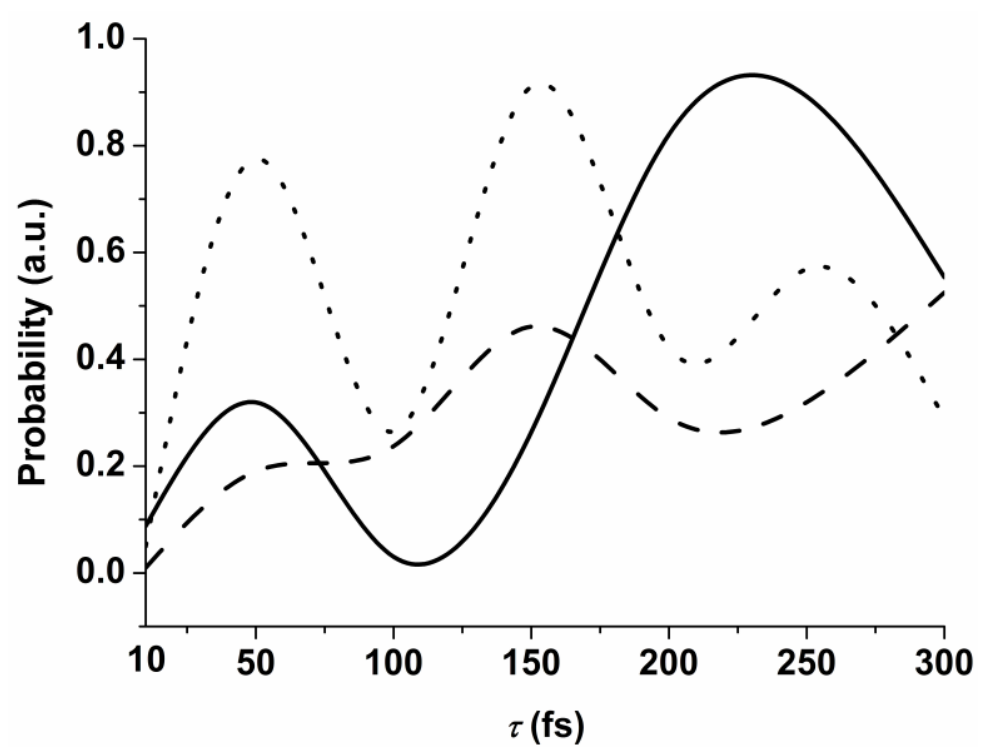

Fig. 6. The population in the excited state $v s$. pulse width. The solid, dashed line, and dotted lines correspond to pump intensity $I_{1}=5.0 \times 10^{11} \mathrm{~W} / \mathrm{cm}^{2}, 5.0 \times 10^{12} \mathrm{~W} / \mathrm{cm}^{2}$, and $5.0 \times 10^{13} \mathrm{~W} / \mathrm{cm}^{2}$, respectively. Other laser parameters are the same as those in Fig. 3.

Fig. 6 shows the population of the excited state $v s$. pulse width at three pump intensities. The population in the excited state changes periodically with increasing pulse width. The pulse width can lead to the different population left in each state by changing pulse area [19]. The change frequency increases with increasing pump intensity, which is consistent with the expectation in Ref. [19].

\section{4. conclusions}

The effect of the pulse width on photoelectron energy spectra, wave packet motion and state populations of the three-level ladder $K_{2}$ molecule driven by pump-probe pulses were investigated via employing the time-dependent wave packet method. With delay time $0 \mathrm{fs}$, the photoelectron spectra show double splitting at longer pulse widths (30 fs , $60 \mathrm{fs}$, and $80 \mathrm{fs}$ ), and no splitting at short pulse width (10 
fs). The photoelectron spectra show double splitting with symmetric profiles at $30 \mathrm{fs}$, but with asymmetric profiles at $60 \mathrm{fs}$ and $80 \mathrm{fs}$. With delay time $1800 \mathrm{fs}$, the photoelectron spectra show double splitting with asymmetric profiles at $80 \mathrm{fs}$, and no splitting at $10 \mathrm{fs}, 30 \mathrm{fs}$ and $60 \mathrm{fs}$. The height of the single peak in the photoelectron spectra becomes higher, and the energy spread becomes narrower with increasing pulse width. The oscillation period of wave packet increases slightly and the oscillation amplitude decreases with increasing pulse width. The populations in the ground state and excited state change periodically with increasing pulse width and the change frequency increases with increasing pump intensity. The results illustrate that by controlling laser pulse width, the selective distribution of state populations can be obtained, which provides the foundation of light manipulation of molecular processes experimentally.

\section{Acknowledgments}

This work was supported by the National Science Foundation of China under Grant No.11447020, the Natural Science Foundation of Hunan province under Grant No. 2015JJ3104, and the Research Foundation of Education Bureau of Hunan province under Grant No. 15B204.

\section{References}

[1] C. Meier, V. Engel, Interference structure in the photoelectron spectra obtained from multiphoton ionization of $\mathrm{Na}_{2}$ with a strong femtosecond laser pulse, Phys. Rev. Lett. 73 (1994) $3207-3210$

[2] W.H. Hu, K.J. Yuan,Y.C. Han, C.C. Shu, S.L. Cong, Autler-Townes splitting in photoelectron spectrum of three-level $\mathrm{Li}_{2}$ molecule in ultrashort pulse laser fields, Chin. Phys. Lett. 24 (2007) $1556-1559$

[3] W.H. Hu, K.J. Yuan, Y.C. Han, C.C. Shu, S.L. Cong, Three-peak Autler-Townes splitting in the photoelectron spectrum of $\mathrm{Li}_{2}$ molecules caused by femtosecond laser pulses, Int. J. Quantum Chem. 110 (2010) 1224-1234.

[4] H.B. Yao, Y.J. Zheng, Quantum control of a molecular system in an intense field via the 
selective population of dressed states, Phys. Chem. Chem. Phys. 13 (2011) 8900-8907.

[5] C.C. Qin, H.S. Zhai, X.Z. Zhang, Y.F. Liu, Coherent control of Autler-Townes splitting in photoelectron spectroscopy: the effect of laser intensity and laser envelope. Bull. Korean. Chem. Soc. 35 (2014) 3294-3298.

[6] W. Guo, X.Q. Lu, X.L. Wang, H.B. Yao, Wavelength dependence of Autler-Townes splitting in a four-level ladder molecular system, EPL 108 (2014) 53002.

[7] Z.G. Sun, N.Q. Lou, Autler-Townes splitting in the moltiphoton resonance ionization spectrum of molecules produced by ultrashort laser pulses, Phys. Rev. Lett. 91 (2003) 023002.

[8] K.J. Yuan, Z.G. Sun, S.L. Cong, N.Q. Lou, Molecular photoelectron spectrum in ultrashort laser fields: Autler-Townes splitting under rotational and aligned effects, Phys. Rev. A 74 (2006) 043421.

[9] K.J. Yuan, Z.G. Sun, S.L. Cong, S.M. Wang, J. Yu, N.Q. Lou, Chem. Phys. 316 (2005) $245-252$.

[10] K.J. Yuan, S.M. Wang, S.L. Cong, Controlling population transfer and dissociation rate of $\mathrm{Na}_{2}$ molecules with Ultra-Short intense laser pulses. Chin. Phys. Lett. 22 (2005) 2534-2537.

[11] Y.F. Liu, R.Q. Liu, J.X. Ding, Theoretical investigation of femtosecond-resolved photoelectron spectra of the $\mathrm{Li}_{2}$ molecule, Chin. Phys. Lett. 26 (2009) 073301.

[12] Y.F. Liu, R.Q. Liu, J.X. Ding, Study on wave packet dynamics of $E^{1} \Sigma_{g}^{+}$state of $\mathrm{Li}_{2}$ with femtosecond-resolved photoelectron spectra, Chin. Phys. B 19 (2010) 033301.

[13] X. Han, W.S. Zhan, S. Wang, J.B. Zai, H.P. Dang, Quantum control of dresses state population for $\mathrm{Li}_{2}$ molecules by intense femtosecond laser pulses, Laser Phys. 26 (2016) 026001

[14] H.B. Yao, Y.J. Zheng, Autler-Townes splitting in photoelectron spectra of $\mathrm{K}_{2}$ molecule, Chin. Phys. B 21 (2012) 023302.

[15] H.B. Yao, W.L. Li, J. Zhang, M. Peng, Quantum control of $\mathrm{K}_{2}$ molecule in an intense laser field: selective population of dressed states, Acta Phys. Sin. 63 (2014) 178201.

[16] X.Q. Lu, W. Guo, Field dependence of state populations in pump-probe pulses, Optik 127 (2016) 5200-5203.

[17] M. Zhang, T.T. Tang, C.M. Zhang, Theoretical study of the influence of femtosecond 
pump-probe pulse on the photoionization of NaLi molecule, Acta Phys. Sin. 63 (2014) 023302 .

[18] J. Wang, F. Liu, D.G. Yue, J. Zhao, Y. Xu, Q.T. Meng, W.K. Liu, Influence of laser fields on the vibrational population of molecules and its wave-packet dynamical investigation, Chin. Phys. B, 19 (2010) 123301.

[19] Q.T. Meng, X.G. Liu, Q.G. Zhang, K.L. Han, Time-dependent wavepacket approach to the influence of intense fields on the population of molecular excited states, Chem. Phys. 316 (2015) 93-98.

[20] H. Schwoerer, R. Pausch, M. Heid, V. Engel, W. Kiefer, Femtosecond time-resolved two-photon ionization spectroscopy of $\mathrm{K}_{2}$, J. Chem. Phys. 107 (1997) 9749-9754.

[21] H. Zhang, K.L. Han, Y. Zhao, G.Z. He, N.Q. Lou, A real time dynamical calculation of $\mathrm{H}_{2}^{-}$ photodissociation, Chem. Phys.Lett. 271 (1997) 204-208.

[22] T.S. Chu, Y. Zhang, K.L. Han, The time-dependent quantum wave packet approach to the electronically nonadiabatic processes in chemical reactions, Int. Rev. Phy. Chem. 25 (2006) 201-235.

[23] Y.J. Li, W.Y. Jiang, Y.G. Khait, M.R. Hoffmann, Theoretical study of the phorodissociation of $\mathrm{Li}_{2}^{+}$in one-color intense laser fields, J. Chem. Phys. 134 (2011) 174108. 